\title{
ANÁLISE MULTIDIMENSIONAL DO DESENVOLVIMENTO RURAL NOS MUNICÍPIOS CEARENSES E PERNAMBUCANOS
}

José Ediglê Alcantara Moura ${ }^{1}$ Eliane Pinheiro de Sousa ${ }^{2}$

Resumo: Este estudo mensura o Índice de Desenvolvimento Rural (IDR) nos municípios cearenses e pernambucanos mediante análise fatorial e relaciona este índice com o Produto Interno Bruto (PIB) agrícola e a população rural por meio da correlação de Pearson. A pesquisa busca responder se os municípios com maiores PIBs agrícolas e população rural estão associados com os maiores IDR. Usaram-se dados majoritariamente dos Censos Demográfico (2010) e Agropecuário (2006). Os resultados revelaram expressiva heterogeneidade no desenvolvimento rural, em que, dos 330 municípios analisados, $56,36 \%$, obtiveram IDR baixo ou muito baixo e apenas 4,24\% atingiram IDR muitíssimo alto. Ademais, as variáveis PIB agrícola e população rural obtiveram correlação forte e moderada com o IDR, nessa ordem.

Palavras-chave: Desenvolvimento rural. Análise fatorial. Correlação de Pearson. Ceará. Pernambuco.

\section{MULTIDIMENSIONAL ANALYSIS OF RURAL DEVELOPMENT IN THE MUNICIPALITIES OF CEARÁ AND PERNAMBUCO}

Abstract: This study measures the Rural Development Index (RDI) in the municipalities of Ceará and Pernambuco through factor analysis and relates this index to the agricultural Gross Domestic Product (GDP) and rural population through Pearson's correlation. This research effort is intended to clarify whether the municipalities with the highest agricultural GDPs and rural populations are associated with the highest RDI indices. It was mostly based on data from the demographic and agricultural censuses of 2010 and 2006, respectively. The results showed that rural development is marked by significant heterogeneity, as $56.36 \%$ of the 330 municipalities analyzed had a low or very low RDI index and only $4.24 \%$ of them recorded a very high RDI index. Furthermore, a strong and moderate correlation with the RDI index was recorded for the agricultural GDP and rural population variables, respectively.

Keywords: Rural development. Factor analysis. Pearson correlation. Ceará. Pernambuco.

\section{ANÁLISIS MULTIDIMENSIONAL DEL DESARROLLO RURAL EN LOS MUNICIPIOS DE CEARÁ Y PERNAMBUCO}

Resumen: Este estudio mensura el Índice de Desarrollo Rural (IDR) en los municipios de Ceará y de Pernambuco mediante el análisis factorial y relaciona este índice con el Producto Interno Bruto (PIB) agrícola y la población rural por medio de la correlación de Pearson. La investigación busca responder si los municipios con mayores PIBs agrícolas y población rural se asocian a los mayores IDR. Se han usado mayoritariamente los datos de los Censos Demográfico (2010) y Agropecuario (2006).

\footnotetext{
1 Universidade Federal do Ceará, Departamento de Economia Agrícola, Fortaleza, Brasil, edigle.economia@gmail.com, https://orcid.org/0000-0003-1285-7717

2 Universidade Regional do Cariri, Departamento de

Economia, Crato, Brasil,
} pinheiroeliane@hotmail.com, https://orcid.org/0000-0003-4088-0754 
Palabras clave: Desarrollo rural. Análisis factorial. Correlación de Pearson. Ceará. Pernambuco.

\section{Introdução}

As estruturas socioeconômicas que caracterizam o Nordeste brasileiro fundamentam-se em relações de produção atrasadas em comparação àquelas vigentes nas áreas mais dinâmicas do país mediante concentração da riqueza e da renda gerada e pela aglutinação de grandes contingentes populacionais em atividades de baixíssima produtividade, insuficiente até mesmo para assegurar os meios de subsistência básicos para as famílias, principalmente nas zonas rurais (CANO, 2011; FURTADO, 1980). No caso dos estados do Ceará e de Pernambuco, o desenvolvimento em uma perspectiva multidimensional encontra significativos pontos de estrangulamento, como alta taxa de analfabetismo rural e concentração fundiária, somados aos gargalos naturais decorrentes das secas (ARAÚJO, 2000).

Segundo o Departamento de Informática do Sistema Único de Saúde (DATASUS, 2010), as taxas de analfabetismo da população rural cearense e pernambucana são, respectivamente, 32,2\% e 33,5\%, estando acima da média nordestina (32,0\%) e nacional (22,9\%). Assim, para Helfand e Pereira (2012), o analfabetismo condiciona as demais estratégias de combate à pobreza e a própria eficácia das ações para inserção produtiva e emancipação dos pobres rurais. Ademais, restringe a opção de migrar, já que este grupo enfrenta maior dificuldade para se inserir na economia urbana, mesmo em atividades informais e/ou na construção civil tradicional absorvedora de trabalho não qualificado.

$\mathrm{Na}$ visão de Grisa e Schneider (2015), o acesso à educação formal é um instrumento para mitigação da pobreza rural. Além disso, as transferências de renda condicionada, aposentadorias rurais e/ou à concessão de crédito aos pequenos agricultores familiares, como: Garantia Safra, Programa Seguro da Agricultura Familiar (SEAF), Programa de Garantia de Preços Mínimos da Agricultura Familiar (PGPAF), Programa de Aquisição de Alimentos (PAA) e Programa Nacional de Alimentação Escolar (PNAE) ajudam, pelo menos a curto e médio prazo, no bemestar das famílias alocadas em áreas rurais.

Por outro lado, conforme dados do Censo Agropecuário de 2006, divulgados pelo IBGE (2010a), percebe-se que o Ceará possui 381.017 estabelecimentos agropecuários, ocupando 1.145.990 trabalhadores, formados preponderantemente por pequenas e médias propriedades, em que 67,57\% têm menos de 10 hectares, 
enquanto que Pernambuco possui 304.790 estabelecimentos agropecuários, alocando 1.324.980 trabalhadores, sendo que 57,89\% têm menos de 10 hectares. Tais dados indicam um perfil de concentração fundiária nestes estados.

A absorção da mão de obra no meio rural está longe de apresentar uma estrutura socioeconômica virtuosa e dinâmica; ao contrário, é reflexo das distorções estruturais, uma vez que a ocupação no meio rural reflete, de um lado, sistemas produtivos dominantes e, do outro, sistemas de baixa produtividade do trabalho e mais intensivo em mão de obra (BALSADI, 2009).

Embora este cenário ainda persista, o setor agrícola do Nordeste brasileiro vem passando por uma reorganização produtiva, apontando para um processo de melhoria da competitividade e consolidação de uma estrutura produtiva mais adaptada ao ambiente econômico de maior concorrência, com resultados expressivos para a agropecuária, mediante a inserção de polos de fruticultura irrigada, como nos municípios de Russas, Limoeiro do Norte e Morada Nova localizados na mesorregião Jaguaribe no Ceará ${ }^{3}$ e Lagoa Grande, Santa Maria da Boa Vista e Petrolina, na mesorregião do São Francisco em Pernambuco4.

Tais condições mostram a visão do desenvolvimento rural associada ao desenvolvimento agrícola devido ao fato de que as proposições do modelo adotado têm elevado fortemente a produção agrícola, porém não resolvem o problema da pobreza rural e nem geram um padrão de vida rural que atendam às necessidades dos que vivem no campo (KAGEYAMA, 2004). Nesse contexto, torna-se necessário apresentar as diferenças entre desenvolvimento agrícola, que corresponde às condições da produção agrícola e/ou agropecuária com suas características no sentido produtivo, identificando suas tendências em um período de tempo e, desenvolvimento rural, que se refere a uma ação articulada que induz mudanças em um dado ambiente rural, reconhecendo que, nas últimas décadas, ocorreu um crescimento nas ocupações não agrícolas no meio rural (NAVARRO, 2001).

No tocante à participação relativa da população rural nordestina, houve um acréscimo em relação ao país de 46,4\% para 47,8\%, entre 2000 e 2010 . Somente

\footnotetext{
3 Segundo IBGE (2010b), estes municípios cearenses destacam-se pelas maiores participações do setor agropecuário no Produto Interno Bruto: Russas (13,46\%), Limoeiro do Norte (10,67\%) e Morada Nova $(9,54 \%)$. No tocante à produção agrícola destes municípios, em termos absolutos, destacam-se Limoeiro do Norte (78.428 ton.), Russas (53.786 ton.) e Morada Nova (23.754 ton.).

4 De acordo com IBGE (2010a), estes municípios pernambucanos destacam-se pelas maiores participações do setor agropecuário no Produto Interno Bruto: Lagoa Grande $(55,12 \%)$, Santa Maria da Boa Vista $(35,83 \%)$ e Petrolina $(21,27 \%)$. No que concerne à produção agrícola destes municípios, em termos absolutos, destacam-se Petrolina (464.168 ton.), Santa Maria da Boa Vista (125.750 ton.) e Lagoa Grande (66.226 ton.).
} 
os estados da Bahia, Maranhão, Ceará e Pernambuco detinham mais de um terço dos habitantes em áreas rurais do Brasil em 2010 (IBGE, 2010b).

Segundo dados do Censo Demográfico do IBGE (2010b), os municípios cearenses com população rural superior à urbana participam com $43 \%$ da população rural estadual (901.428 habitantes), enquanto os municípios pernambucanos participam com aproximadamente um terço do total de habitantes do estado residentes na zona rural, atingindo 32,40\% (563.812 indivíduos), necessitando, assim, de estudos que viabilizem os determinantes para aferição de melhores níveis de desenvolvimento rural para 2010.

Diante de tais aspectos, questiona-se: os municípios cearenses e pernambucanos com maiores PIBs agrícolas e população rural estão associados com maior nível de desenvolvimento rural? Estudos desta natureza podem contribuir para o aprimoramento e focalização de políticas públicas, ao identificar os principais gargalos que impedem o alcance do bem-estar da população cearense e pernambucana em áreas rurais. Portanto, este trabalho se propõe mensurar o Índice de Desenvolvimento Rural (IDR) nos municípios cearenses e pernambucanos para o ano de 2010 e relacionar o IDR com o Produto Interno Bruto (PIB) agrícola e a população rural.

\section{Revisão bibliográfica}

A literatura referente ao desenvolvimento rural demonstra que essa temática tem sido tratada em múltiplos recortes geográficos por meio da construção de um Índice de Desenvolvimento Rural (IDR). Dentre estes, destacam-se os trabalhos de Stege (2011) e Kageyama (2008). Em termos regionais, Polastrini, Martins e Tredezini (2015) centram a discussão nas regiões Nordeste e Centro-Oeste. Em termos de municípios, tem-se os estudos de Alves (2012) para Goiás; Melo e Parré (2007) para o Paraná; e Kageyama (2004) para São Paulo. Quanto ao Nordeste, o estudo de Lima e Sousa (2017) aborda uma amostra de municípios pernambucanos e baianos, e o trabalho de Sales et al. (2017) considera os municípios cearenses.

Segundo Lemos (1988), o desenvolvimento não se caracteriza de forma igualitária em todas as regiões, em que as sinergias agem em pontos isolados desencadeando concentrações espaciais. Para alcançar o desenvolvimento, Jesus (2013) argumenta que é preciso formular políticas públicas que as tornem realidade, levando em conta as características de cada região. 
Kageyama (2004) utilizou o Censo Demográfico de 1991 e 2000 e o Censo Agropecuário de 1995, a fim de propor um índice para o desenvolvimento rural nos municípios paulistas. Os resultados mostraram que os municípios com IDR alto encontraram-se mais de $50 \%$ da população rural estadual, enquanto no nível médio, que são cerca da metade dos municípios, estão aproximadamente $31 \%$ da população rural, sendo que apenas $16,7 \%$ desse contingente vivem em municípios de baixo desenvolvimento. A partir desta obra, vários estudos foram intensificados sobre esse tema, utilizando abordagens metodológicas relativas aos índices parciais, como Lima e Sousa (2017) e Kageyama (2008); ou análise fatorial, como Sales et al. (2017); Pinto e Coronel (2016); Stege (2011) e Melo e Parré (2007), buscando a multidimensionalidade do desenvolvimento proposto.

Stege (2011) elaborou o IDR para as 558 microrregiões brasileiras para 2008. Verificou-se que 15 microrregiões apresentaram desenvolvimento rural extremamente alto; 56, alto grau de desenvolvimento; 202 com grau médio alto, e 285 alocadas com nível regular de desenvolvimento, sendo representado por $51,07 \%$ do total das microrregiões. Porém, apenas $12,7 \%$ delas possuem um nível acima da classificação médio alto, o que demonstra a heterogeneidade estrutural nos territórios rurais brasileiros.

Para as microrregiões do Nordeste brasileiro, Stege (2011) constatou que Barreiras (BA) e Maceió ( $A L)$ obtiveram maiores níveis de desenvolvimento rural dentre as verificadas na região. Os fatores responsáveis que contribuíram para a primeira foram: produtividade dos fatores de produção, renda agrícola, preservação ambiental e crédito rural. Para a segunda, o seu grau de desenvolvimento rural foi proporcionado pela produtividade dos fatores de produção, renda agrícola, qualidade de vida nos domicílios rurais e diversidade da atividade agropecuária. Essas informações são confirmadas por meio dos dados do Censo Demográfico (IBGE, 2010b) que revelam, do total de sete municípios da microrregião de Barreiras (BA), três (Baianópolis, Riachão das Neves e São Desidério) apresentaram, em média, $63,92 \%$ da população total alocada nas zonas rurais. Ademais, são municípios cujo PIB agrícola supera as demais participações nos setores da atividade econômica, com, 48,87\%, 69,11\% e 78,99\%, respectivamente.

Os resultados de Stege (2011) estão consistentes com as proposições de Kageyama (2008) que discorre sobre os conceitos e aplicações ao caso brasileiro dos determinantes do desenvolvimento rural, afirmando que os fatores que permitem a sua aferição não se restringem ao crescimento econômico, medido pelo produto e 
renda per capita, uma vez que esse fenômeno é compreendido como um processo que envolve a dimensão econômica, sociocultural, político, institucional e ambiental.

Polastrini, Martins e Tredezini (2015) seguiram a abordagem metodológica de Kageyama (2004), e analisaram o IDR dos Consórcios de Segurança Alimentar e Desenvolvimento Local (CONSAD) das regiões Centro-Oeste e Nordeste. Os resultados indicaram, que, dos 12 CONSADS analisados do Nordeste, apenas dois estão na categoria alta, o de Itaparica do Pernambuco que obteve maior renda domiciliar, e o Sertão do São Francisco em Sergipe, que se destacou pela proporção de domicílios rurais com infraestrutura de acesso à água. Somente um está na categoria média, que é Baturité no Ceará, que apresentou melhor média de anos de estudos na proporção de crianças de 7 a 14 anos na escola, enquanto os demais estão na categoria baixa. Para o Centro-Oeste, dos cinco CONSADS estudados, três pertencentes ao estado de Mato Grosso do Sul estão classificados na categoria média, e o restante se classifica como alta.

Em termos municipais, Alves (2012) construiu o IDR para os municípios goianos. O autor focou apenas na dimensão econômica e obteve elevada heterogeneidade com predominância de regiões com baixos níveis de desenvolvimento rural. Em conformidade com Melo e Parré (2007), indicadores de renda sintetizam as condições necessárias, mas não suficientes para mensuração do desenvolvimento rural.

O grande número de municípios com pífias condições de bem-estar no meio rural é reforçado por Melo e Parré (2007), que mensuraram o IDR dos municípios paranaenses para 2000. Esses resultados convergem com os estudos de Stege (2011) para as microrregiões sulistas, mostrando que o número de alunos matriculados, a utilização de energia elétrica no meio rural e o acesso ao crédito são as variáveis que expressam maior desenvolvimento rural. Não obstante, mais da metade dos municípios paranaenses se encontra em níveis baixos de desenvolvimento rural, indicando forte heterogeneidade nos territórios rurais, sendo também constatada por Kageyama (2004).

Diante dessas considerações, para Pacheco (1998), há "ilhas de produtividade" em diferentes regiões, até mesmo as mais atrasadas, como no Nordeste brasileiro. Nessa perspectiva, Lima e Sousa (2017) mensuraram o IDR para oito municípios da Região Integrada de Desenvolvimento (RIDE) do Polo Petrolina (PE) e Juazeiro (BA). Para tanto, foram aferidos o Índice de População (IPOP), Índice de Bem-Estar Social (IBES), Índice de Desenvolvimento Econômico 
(IDE) e Índice de Meio Ambiente (IMA). Os resultados mostraram disparidades, em que Petrolina e Juazeiro se configuraram com alto IDR. Por outro lado, metade dos municípios dessa região foi classificada com IDR baixo, porém acima de outras áreas do Nordeste brasileiro.

Os dados do Censo Demográfico (IBGE, 2010b) revelam que, do total dos oito municípios da RIDE destacada, Lagoa Grande, Orocó e Santa Maria da Boa Vista, em Pernambuco, e Curaçá, na Bahia, possuem população rural superior à urbana, e participam, em média, com $61 \%$ do total de indivíduos alocados no meio rural. Vale salientar que Lagoa Grande (PE) é o único município em que a participação do PIB agrícola (55,12\%) supera os demais setores da economia.

Sales et al. (2017) analisaram o IDR dos municípios cearenses para 2000 e 2010. Verificaram que as mesorregiões Jaguaribe, Centro-Sul e Noroeste obtiveram os maiores índices, sendo que os determinantes do desenvolvimento rural foram convergentes com Pinto e Coronel (2016), em que a proporção de pessoas que frequentavam o ensino básico e a proporção da população rural no meio rural impacta positivamente o bem-estar da população rural.

Portanto, tendo em vista que os estudos realizados sobre desenvolvimento rural em Pernambuco investigaram apenas os municípios da Região Integrada de Desenvolvimento de Petrolina (PE) e Juazeiro (BA), como é o caso do trabalho realizado por Lima e Sousa (2017), este estudo inova neste sentido. Além de ampliar a amostra dos municípios pernambucanos considerados, também apresenta uma diferenciação em termos do método analítico empregado. No caso do Ceará, há investigações dessa natureza em todos os municípios (SALES et al., 2017), porém não abrangeu as variáveis de cunho socioeconômico, ambiental e demográfico presentes neste trabalho. Ademais, outra contribuição deste estudo em relação à literatura é que foram investigados se o PIB agrícola e a população rural estão correlacionados com o desenvolvimento rural.

\section{Metodologia}

\section{Métodos analíticos}

Para identificar os determinantes do desenvolvimento rural para os municípios cearenses e pernambucanos e mensurar o Índice de Desenvolvimento Rural (IDR), foi utilizada a técnica estatística multivariada de análise fatorial pelo método dos componentes principais. Para Rezende e Parré (2004), este 
instrumental consiste em reduzir um grande conjunto de variáveis em um pequeno número de fatores em função de suas correlações, possibilitando a criação de indicadores e facilitando a interpretação dos dados.

$X_{i}=\alpha_{i 1} F_{1}+\alpha_{i 2} F_{2}+\ldots+\alpha_{i m} F_{m}+\varepsilon_{i}(1)$, em que: $X_{i}=i$-ésimo escore da variável original; $i$ $=$ número de variáveis analisadas, sendo $i=1,2, \ldots, p$; $a_{i j}=$ carga fatorial $i$ no fator $j$; $F_{j}=$ fator aleatório comum; $j=$ número de fatores gerados, sendo $j=1,2, \ldots, m ; \varepsilon_{i}=$ fatores específicos, componente aleatório especifico para cada componente.

Nesta pesquisa, optou-se em estudar os determinantes do desenvolvimento rural nos municípios cearenses e pernambucanos, não permitindo que a análise fatorial seja realizada individualmente para cada estado, pois os fatores obtidos em cada unidade federativa apresentariam valores diferentes e inviabilizariam verificar 0 comportamento do desenvolvimento rural, considerando o conjunto dos municípios deste estudo. Portanto, na análise fatorial, foram agregadas as observações das variáveis componentes do IDR nos municípios dos dois estados observados para o ano de 2010, podendo ser expresso em termos matriciais pela expressão (2).

$M=\left[\begin{array}{l}M_{1} \\ M_{2}\end{array}\right]$ (2), em que: M representa a matriz de dimensão $330 \times 15$, sendo que 330 corresponde o conjunto dos municípios cearenses (172) e pernambucanos (158) considerados neste estudo para 2010 e 15 referem-se às variáveis utilizadas na mensuração do IDR; e $M_{1}$ e $M_{2}$ representam, respectivamente, a matriz dos municípios cearenses e pernambucanos, em 2010, com as variáveis do IDR.

Neste trabalho, a extração dos fatores iniciais foi considerada pela Análise dos Componentes Principais, que mostrou uma combinação linear das variáveis observadas, buscando maximizar a variância total explicada. A escolha do número de fatores se deu mediante a raiz latente, em que se selecionou o número de fatores em função dos valores próprios acima de um (eigenvalues), que mostraram quanto cada fator conseguirá explicar da variância total (MINGOTI, 2005). Para a rotação dos fatores, empregou-se o método Varimax, que objetiva maximizar a variação entre os pesos de cada componente principal, mantendo-se a ortogonalidade entre eles. Em seguida, calculou-se a matriz dos coeficientes fatoriais, obtida a partir do produto da matriz transposta das cargas fatoriais com a inversa da matriz de correlações simples entre as variáveis utilizadas na análise (FÁVERO et al., 2009).

Quanto ao cálculo do IDR, este trabalho se baseou nos estudos de Stege (2011), Pinto e Coronel (2016), e Begnini e Almeida (2016), em que tal índice pode ser indicado pela soma dos escores fatoriais padronizados, obtidos pela análise 
fatorial, ponderados pelas respectivas parcelas de explicação da variância total dos dados de cada fator. Matematicamente, o IDR pode ser dado pela equação (3):

$I D R=\sum_{j=1}^{k}\left(\frac{\lambda_{j}}{\sum_{j=1}^{k} \lambda_{j}} F P_{j i}\right), \nabla \lambda \geq 1$ (3), em que: IDR é o Índice de Desenvolvimento Rural; $\lambda_{j}$ é o percentual da variância explicada pelo fator $\mathrm{j} ; \mathrm{k}$, número de fatores escolhidos; $F P_{j i}$ é o escore fatorial, padronizado pelo município i, do fator j, que, com base nesses estudos referenciados, pode ser representado pela equação (4):

$F P_{j i}=\frac{F_{j}-F_{\min }}{F_{\max }-F_{\min }}(4)$, em que: $F_{\min }$ é o escore fatorial mínimo do fator j; e $F_{\max }$ é o escore fatorial máximo do fator j. Ademais, $F P_{j i}$ está disposto de tal forma que o pior resultado é 0 e o melhor é 1.

Com o intuito de classificar o desenvolvimento rural em grupos, empregou-se a denominação utilizada por Begnini e Almeida (2016); Pinto e Coronel (2016); Stege (2011) e Melo e Parré (2007), a partir do valor médio do índice. A escala utilizada é relativa, tendo seus valores baseados na média. Foram classificados com Índice de Desenvolvimento Rural muitíssimo alto (MMA) aqueles municípios que apresentaram resultados com três desvios-padrão acima da média; muito alto (MA), os municípios com dois e três desvios-padrão acima da média; alto $(A)$, os municípios com valores entre um e dois desvios-padrão acima da média; médio $(\mathrm{M})$, os municípios que apresentaram resultados entre a média e um desvio- padrão acima da média; baixo (B), os municípios com valores no intervalo entre e média e um desvio-padrão abaixo da média; muito baixo (MB), os municípios com um e dois desvios-padrão abaixo da média; e, por fim, muitíssimo baixo (MMB), os municípios com dois desvios-padrão abaixo da média. A interpretação do IDR é que, quanto maior o seu valor, maior é o nível de desenvolvimento rural do recorte geográfico analisado. Essa classificação está exposta no Quadro 01.

Quadro 01- Classificação do Índice de Desenvolvimento Rural (IDR)

\begin{tabular}{|c|c|c|}
\hline IDR & Sigla & Desvios-padrão $(\delta)$ em torno da média \\
\hline Muitíssimo alto & MMA & MMA $\geq(\mathrm{M}+3 \delta)$ \\
\hline Muito alto & MA & $(\mathrm{M}+2 \delta) \leq M A<(M+3 \delta)$ \\
\hline Alto & $\mathrm{A}$ & $(\mathrm{M}+1 \delta) \leq \mathrm{A}<(\mathrm{M}+2 \delta)$ \\
\hline Médio & $\mathrm{M}$ & $(\mathrm{Média}) \leq \mathrm{M}<(\mathrm{M}+1 \delta)$ \\
\hline Baixo & $\mathrm{B}$ & $(\mathrm{M}-1 \delta) \leq \mathrm{B}<($ Média $)$ \\
\hline Muito baixo & $\mathrm{MB}$ & $(\mathrm{M}-2 \delta) \leq \mathrm{MB}<(\mathrm{M}-1 \delta)$ \\
\hline
\end{tabular}


\begin{tabular}{|l|l|r} 
Muitíssimo baixo & MMB & MMB $\leq(M-2 \delta)$ \\
\hline
\end{tabular}

Fontes: Begnini e Almeida (2016); Pinto e Coronel (2016); Stege (2011); Melo e Parré (2007).

Além de mensurar o IDR para os municípios cearenses e pernambucanos aplicando o método de análise fatorial, esse trabalho determinou o teste Alfa de Cronbach para analisar a confiabilidade dos resultados.

Para relacionar o Índice de Desenvolvimento Rural (IDR) com o PIB agrícola e a população rural, empregou-se o coeficiente de correlação de Pearson. Segundo Triola (2013), este coeficiente é caracterizado por um índice adimensional (r) com valores situados ente -1,0 e 1,0, o que reflete a intensidade da relação linear entre dois conjuntos de dados.

A aplicação deste método tomou como base os estudos de Dalchiavon e Carvalho (2012), que estabeleceram uma correlação linear e espacial entre os componentes de produção e produtividade da soja; Santini, Oliveira e Pigatto (2010), que analisaram a relação das variáveis preço e produção da mandioca tipo indústria no estado de São Paulo; e Silva e Lima (2015), que estabeleceram a relação entre as variáveis educacionais, renda e pobreza. Assim, o coeficiente de correlação de Pearson ( $r$ ) pode ser expresso pela equação (5):

$$
r=\frac{\sum_{i}\left(x_{i}-\bar{x}\right)\left(y_{i}-\bar{y}\right)}{\sqrt{\sum_{i}\left(x_{i}-\bar{x}\right)^{2} \sum_{i}\left(y_{i}-\bar{y}\right)^{2}}}(5) \text {, em que: } r \text { é o valor da correlação, } x_{i} \text { e } y_{i} \text { são as }
$$

variáveis estudadas, seguidas dos seus valores médios, respectivamente. De acordo com Bisqueira, Sarriera e Martínez (2004, p. 47), a classificação da correlação pode ser mostrada no Quadro 02.

Quadro 02- Classificação da correlação de Pearson

\begin{tabular}{|c|c|}
\hline Intervalos & Tipo de correlação \\
\hline$-1,00$ & Negativa perfeita \\
\hline
\end{tabular}




\begin{tabular}{|c|c|}
{$[-1,00 ;-0,80[$} & Negativa muito alta \\
\hline$[-0,80 ;-0,60[$ & Negativa alta \\
\hline$[-0,60 ;-0,40[$ & Negativa moderada \\
\hline$[-0,40 ;-0,20[$ & Negativa baixa \\
\hline$[-0,20 ; 0,00[$ & Negativa muito baixa \\
\hline 0,00 & Nula \\
\hline$] 0,00 ; 0,20]$ & Positiva muito baixa \\
\hline$] 0,20 ; 0,40]$ & Positiva baixa \\
\hline$] 0,40 ; 0,60]$ & Positiva moderada \\
\hline$] 0,60 ; 0,80]$ & Positiva alta \\
\hline$] 0,80 ; 1,00[$ & Positiva muito alta \\
\hline 1,00 & Positiva perfeita \\
\hline
\end{tabular}

Fonte: Bisqueira, Sarriera e Martínez (2004).

\section{Base de dados e descrição das variáveis}

Os dados empregados para este estudo são de origem secundária e coletados a partir do Censo Demográfico (2010), Censo Agropecuário (2006) e Produção Agrícola Municipal (2010), alocados no Banco de Dados Agregados do Sistema IBGE de Recuperação Automática (SIDRA), publicados pelo Instituto Brasileiro de Geografia e Estatística (IBGE). Utilizaram-se também as bases de dados do Departamento de Informática do Sistema Único de Saúde (DATASUS, 2010) e da Relação Anual de Informações Sociais do Ministério do Trabalho e Emprego (RAIS/MTE, 2010). A limitação da disponibilidade de dados inibe contemplar todos os aspectos inerentes ao processo de desenvolvimento. A fim de mensurar o grau de desenvolvimento do meio rural dos municípios ${ }^{5}$ cearenses e pernambucanos, foram selecionadas variáveis ${ }^{6}$ que permitam abranger as dimensões socioeconômica, demográfica e ambiental (Quadro 3).

\footnotetext{
${ }^{5}$ As áreas de estudo deste trabalho contemplaram 330 do total de 369 municípios cearenses e pernambucanos, sendo removidos da análise os municípios de Chaval, Eusébio, Fortaleza, Itaitinga, Martinópole, Miraíma, Orós, Pedra Branca, Potengi, Quixeré, Solonópole e Umari para o estado do Ceará, e Bonito, Cabo de Santo Agostinho, Camagaribe, Camocim de São Félix, Carnaubeira da Penha, Chã de Alegria, Chã Grande, Cortês, Dormentes, Escada, Fernando de Noronha, Iguaracy, Ingazeira, Itaíba, Paulista, Recife, Ribeirão, Sairé, São José da Coroa Grande, São Lourenço, Sertânia, Tacaracutu, Tamandaré, Toritama, Triunfo, Tuparetama e Venturosa para a unidade federativa de Pernambuco. Essa seleção foi baseada na disponibilidade de dados para todas as variáveis contidas neste estudo.

6 Além dessas trabalhadas neste estudo, testaram-se ainda o número de estabelecimentos agropecuários que contraíram financiamentos, salário médio nominal da agropecuária (em $R \$$ ), produtividade média das lavouras temporárias, produtividade média das lavouras permanentes, número de beneficiários de programas de transferência de renda condicionada, número de ocupados alocados na zona rural com qualificação técnica, número de estabelecimentos agropecuários com recursos hídricos, número de estabelecimentos agropecuários com orientação técnica, número de estabelecimentos agropecuários que usufruíram de financiamentos, área plantada de mandioca e o número de estabelecimentos agropecuários com máquinas e implementos agrícolas, número de domicílios rurais que possuem energia elétrica e número de domicílios rurais que possuem sistema de esgoto e rede pública geral, porém tais variáveis não foram consideradas neste estudo por apresentarem baixas comunalidades.
} 
Quadro 03- Dimensões, variáveis e fontes de dados no ano de 2010 para o Índice de Desenvolvimento Rural (IDR) para os municípios cearenses e pernambucanos

\begin{tabular}{|c|c|c|c|c|}
\hline DIMENSÕES & VARIÁVEIS & DESCRIÇÃO & FONTES & $\begin{array}{c}\text { ESTUDOS } \\
\text { FUNDAMENTADOS }\end{array}$ \\
\hline \multirow{7}{*}{ SOCIOECONÔMICO } & V1 & $\begin{array}{l}\text { Número de domicílios rurais servido } \\
\text { de água proveniente de uma rede } \\
\text { geral de abastecimento (unidades) }\end{array}$ & $\begin{array}{l}\text { Censo } \\
\text { Demográfico do } \\
\text { IBGE (2010) }\end{array}$ & \multirow{7}{*}{$\begin{array}{l}\text { Melo e Parré (2007); } \\
\text { Stege (2011); Begnini } \\
\text { e Almeida (2016) e } \\
\text { Alves (2012) }\end{array}$} \\
\hline & V2 & $\begin{array}{l}\text { Número de domicílios rurais que } \\
\text { possuem lixo coletado por serviço, } \\
\text { empresa pública ou particular } \\
\text { (unidades) }\end{array}$ & $\begin{array}{l}\text { Censo } \\
\text { Demográfico do } \\
\text { IBGE (2010) }\end{array}$ & \\
\hline & V3 & $\begin{array}{l}\text { Número de pessoas alfabetizadas que } \\
\text { estavam alocadas no meio rural }\end{array}$ & $\begin{array}{l}\text { Censo } \\
\text { Demográfico do } \\
\text { IBGE (2010) }\end{array}$ & \\
\hline & V4 & $\begin{array}{lrrr}\text { Valor } & \text { Adicionado } & \text { Bruto } & \text { da } \\
\text { Agropecuária (VAB) } & & \\
\end{array}$ & DATASUS (2010) & \\
\hline & V5 & $\begin{array}{l}\text { Número de vínculos formais de } \\
\text { trabalho no setor agropecuário em } \\
31 / 12 / 2010 \text { (pessoas) }\end{array}$ & RAIS/ MTE (2010) & \\
\hline & V6 & $\begin{array}{l}\text { Número de estabelecimentos } \\
\text { agropecuários que utilizaram técnica } \\
\text { de irrigação (unidades) }\end{array}$ & $\begin{array}{l}\text { Censo } \\
\text { Agropecuário do } \\
\text { IBGE (2006) } \\
\end{array}$ & \\
\hline & V7 & $\begin{array}{l}\text { Valor da produção das lavouras } \\
\text { permanentes (em mil } \mathrm{R} \$ \text { ) }\end{array}$ & $\begin{array}{l}\text { Censo } \\
\text { Agropecuário do } \\
\text { IBGE (2006) }\end{array}$ & \\
\hline \multirow[t]{2}{*}{ DEMOGRÁFICO } & V8 & $\begin{array}{l}\text { Proporção de migrantes (\%) alocados } \\
\text { na zona rural do município }\end{array}$ & $\begin{array}{l}\text { Censo } \\
\text { Demográfico do } \\
\text { IBGE (2010) }\end{array}$ & \multirow{2}{*}{$\begin{array}{l}\text { Kageyama (2004; } \\
\text { 2008) }\end{array}$} \\
\hline & V9 & $\begin{array}{l}\text { Proporção da população rural (\%) em } \\
\text { relação à população total }\end{array}$ & $\begin{array}{l}\text { Censo } \\
\text { Demográfico do } \\
\text { IBGE (2010) }\end{array}$ & \\
\hline \multirow{6}{*}{ AMBIENTAL } & V10 & Área plantada de feijão (ha) & $\begin{array}{l}\text { Produção Agrícola } \\
\text { Municipal do IBGE } \\
\text { (2010) }\end{array}$ & \multirow{6}{*}{$\begin{array}{l}\text { Santos, Ferreira e } \\
\text { Salgado (2017); Pinto } \\
\text { e Coronel (2016) e } \\
\text { Kageyama (2004). }\end{array}$} \\
\hline & V11 & Área plantada de milho (ha) & $\begin{array}{l}\text { Produção Agrícola } \\
\text { Municipal do IBGE } \\
(2010)\end{array}$ & \\
\hline & V12 & $\begin{array}{l}\text { Número de estabelecimentos } \\
\text { agropecuários que utilizaram sistema } \\
\text { de preparação do solo (unidades) }\end{array}$ & $\begin{array}{l}\text { Censo } \\
\text { Agropecuário do } \\
\text { IBGE (2006) }\end{array}$ & \\
\hline & V13 & $\begin{array}{l}\text { Número de estabelecimentos } \\
\text { agropecuários que utilizaram } \\
\text { agrotóxicos (unidades) }\end{array}$ & $\begin{array}{l}\text { Censo } \\
\text { Agropecuário do } \\
\text { IBGE (2006) }\end{array}$ & \\
\hline & V14 & $\begin{array}{l}\text { Número de } \frac{\text { estabelecimentos }}{\text { agropecuários que utilizaram as }} \\
\text { queimadas como prática agrícola } \\
\text { (unidades) }\end{array}$ & $\begin{array}{l}\text { Censo } \\
\text { Agropecuário do } \\
\text { IBGE (2006) }\end{array}$ & \\
\hline & V15 & $\begin{array}{l}\text { Número de estabelecimentos } \\
\text { agropecuários que utilizaram técnica } \\
\text { de adubação (unidades) }\end{array}$ & $\begin{array}{l}\text { Censo } \\
\text { Agropecuário do } \\
\text { IBGE (2006) }\end{array}$ & \\
\hline
\end{tabular}

Fonte: elaborado a partir dos dados do Censo Demográfico (2010); Produção Agrícola Municipal (2010) e Censo Agropecuário (2006), extraídos do banco de dados agregados SIDRA do IBGE. RAIS/MTE (2010) e DATASUS (2010).

\section{Resultados e discussão}

\section{Análise Fatorial}

A partir da matriz de correlações, observam-se elevados coeficientes de correlação para a maioria dos pares de variáveis. Isso evidencia, a priori, que os dados parecem ser adequados para análise fatorial. Outro indício de que a análise fatorial é apropriada se refere à matriz de antiimagem, que revelou baixos coeficientes. O teste de esfericidade de Bartlett mostrou-se significativo a $1 \%$ de probabilidade, com valor de 5.317,54, rejeitando a hipótese nula de que a matriz de 
correlação é uma matriz identidade. Com o intuito de verificar a coesão dos dados, foi calculado o KMO, que apresentou valor de 0,83. Segundo Fávero et al. (2009), quando os valores do KMO pertencem ao intervalo entre 0,80 a 0,90, como é o caso deste estudo, a utilização desta técnica apresenta boa adequação da amostra. Assim, de posse dos resultados dos testes estatísticos, pode-se concluir que o conjunto de dados é adequado para o emprego da análise fatorial.

Quanto à confiabilidade das variáveis, averiguou-se mediante a estimação do Alfa de Cronbach. Para Hair Júnior et al. (2005), este teste varia de 0 a 1, sendo que os valores compreendidos entre 0,60 a 0,70 são considerados o limite inferior de aceitabilidade, de modo que, quanto mais se aproxime de 1, maior a confiabilidade. Analisando os resultados para cada fator, percebe-se que o mais confiável foi a dimensão ambiental $(0,89)$, seguida pela demográfica $(0,84)$ e socioeconômica $(0,62)$. Tais resultados permitem inferir que esses fatores mensuram de forma adequada o desenvolvimento rural nos municípios cearenses e pernambucanos.

Portanto, ao se constatar que esse método analítico foi adequado à base de dados, utilizou-se o método dos componentes principais. Vale destacar, porém, que, em sua versão original, uma variável pode se relacionar com mais de um fator, dificultando a interpretação. Assim, com o intuito de remover esse tipo de problema, adotou-se a rotação ortogonal pelo método Varimax, cujas raízes características da matriz de correlações são valores superiores à unidade e suas respectivas porcentagens da variância total (MINGOTI, 2005).

Os resultados da análise fatorial identificaram três fatores com autovalores maiores que a unidade, considerando o critério da raiz latente, conforme indicado pela Tabela 01. Dessa forma, as dimensões (socioeconômica, ambiental e demográfica) sintetizam as 15 variáveis consideradas, sendo capazes de explicar $76,02 \%$ da variância total dos dados.

Tabela 01- Valores das raízes características e percentual de variância total explicada pela análise fatorial

\begin{tabular}{c|c|c|c}
\hline Fator & Raiz característica & $\begin{array}{c}\text { Variância explicada pelo fator } \\
(\%)\end{array}$ & $\begin{array}{c}\text { Variância acumulada } \\
(\%)\end{array}$ \\
\hline 1 & 4,89 & 32,62 & 32,62 \\
2 & 4,68 & 31,22 & 63,84 \\
3 & 1,83 & 12,18 & 76,02 \\
\hline
\end{tabular}

Fonte: elaborada com base nos resultados da pesquisa.

A Tabela 02 mostra as cargas fatoriais rotacionadas e as comunalidades para os fatores considerados. Neste estudo, as cargas fatoriais com valores absolutos a partir de 0,6 foram destacadas em negrito como indicativo das variáveis mais 
fortemente associadas a um dado fator. Os valores das comunalidades sinalizam que todas as variáveis têm sua variabilidade representada pelos três fatores.

Como se observa, o fator F1 explica a maior variância $(32,62 \%)$ e reflete de forma consistente a dimensão socioeconômica, possuindo correlação positiva e forte com as variáveis $\mathrm{V} 1, \mathrm{~V} 2, \mathrm{~V} 3, \mathrm{~V} 4, \mathrm{~V} 5, \mathrm{~V} 6$ e V7.

No que concerne às variáveis V6 e V3, em conjunto, com sinais positivos e valores elevados conduzem a um maior desempenho econômico do meio rural cearense e pernambucano, estando consistente com a teoria econômica, uma vez que, Coeteris paribus, o acréscimo da tecnologia na agricultura por meio da irrigação, demanda maior produtividade marginal da mão-de-obra, o que pode ser conferido nos trabalhadores com maior instrução formal. Esses resultados foram confirmados por Begnini e Almeida (2016) para os municípios catarinenses, ao apontar a educação rural e a irrigação como determinantes do desenvolvimento rural. A relutância dos trabalhadores rurais em aderir novas tecnologias persiste, sobretudo, pela baixa escolaridade. Dentro dessa perspectiva, Coelho (2009) enfatizou o baixo nível de instrução formal como prejudicial à implantação das tecnologias, causando impactos negativos na gestão dos estabelecimentos rurais.

No tocante às variáveis V4 e V7, percebe-se elevada correlação entre valor adicionado bruto do setor agropecuário e valor da produção das lavouras permanentes. Tal resultado corrobora as evidências de Cardoso e Souza (2000), que ressaltam as lavouras permanentes, especificamente, os ramos da fruticultura irrigada como geradora de sinergias na atividade econômica, ensejando em commodities de maior valor agregado. Para Sabino (2013), as lavouras temporárias se deparam com preços que apresentam níveis de instabilidade e impactam na limitada capacidade de gerar renda monetária a partir dessas atividades agrícolas.

Tabela 02- Cargas fatoriais após a rotação ortogonal e comunalidades, obtidas na análise

\begin{tabular}{|c|c|c|c|c|}
\hline \multirow{2}{*}{ Variáveis } & \multicolumn{3}{|c|}{ Cargas Fatoriais } & \multirow{2}{*}{ Comunalidades } \\
\hline & $\mathrm{F} 1$ & F2 & F3 & \\
\hline $\begin{array}{l}N^{\circ} \text { de domicílios rurais com água de uma rede } \\
\text { geral de abastecimento (V1) }\end{array}$ & 0,83 & 0,33 & 0,05 & 0,80 \\
\hline $\begin{array}{l}\mathrm{N}^{\circ} \text { de domicílios rurais que possuem lixo } \\
\text { coletado por serviço, empresa pública ou } \\
\text { particular (V2) }\end{array}$ & 0,85 & 0,01 & $-0,09$ & 0,73 \\
\hline $\begin{array}{l}N^{\circ} \text { de pessoas alfabetizadas alocadas no meio } \\
\text { rural (V3) }\end{array}$ & 0,66 & 0,62 & 0,05 & 0,82 \\
\hline Valor Adicionado Bruto da Agropecuária (V4) & 0,92 & 0,13 & $-0,04$ & 0,87 \\
\hline $\begin{array}{l}N^{\circ} \text { de vínculos formais de trabalho no setor } \\
\text { agropecuário em } 31 / 12 / 2010 \text { (V5) }\end{array}$ & 0,86 & $-0,10$ & $-0,10$ & 0,76 \\
\hline $\begin{array}{l}N^{0} \text { de estabelecimentos agropecuários com } \\
\text { irrigação (V6) }\end{array}$ & 0,65 & 0,28 & $-0,02$ & 0,50 \\
\hline
\end{tabular}


Valor da produção das lavouras permanentes, expresso em mil reais (V7)

Proporção de migrantes (\%) alocados na zona rural do município (V8)

Proporção da população rural (\%) em relação à população total (V9)

Área plantada de feijão em hectares (V10)

Área plantada de milho em hectares (V11)

$\mathrm{N}^{\circ}$ de estabelecimentos agropecuários com sistema de preparação do solo (V12)

$\mathrm{N}^{\circ}$ de estabelecimentos agropecuários que utilizaram agrotóxicos (V13)

$\mathrm{N}^{\circ}$ de estabelecimentos agropecuários que utilizaram queimadas como prática agrícola (V14)

$\mathrm{N}^{\circ}$ de estabelecimentos agropecuários que utilizaram técnica de adubação (V15)

\begin{tabular}{c|c|c|c}
$\mathbf{0 , 8 9}$ & $-0,01$ & 0,03 & 0,78 \\
$-0,01$ & 0,02 & $\mathbf{0 , 9 5}$ & 0,91 \\
$-0,09$ & 0,16 & $\mathbf{0 , 9 1}$ & 0,91 \\
& & & 0,68 \\
$-0,12$ & $\mathbf{0 , 8 1}$ & 0,04 & 0,63 \\
$-0,13$ & $\mathbf{0 , 7 8}$ & 0,03 & 0,76 \\
0,15 & $\mathbf{0 , 8 5}$ & 0,12 & 0,57 \\
0,29 & $\mathbf{0 , 6 9}$ & 0,08 & 0,86 \\
0,25 & $\mathbf{0 , 8 9}$ & 0,03 & \\
& & & 0,85 \\
0,23 & $\mathbf{0 , 8 9}$ & 0,04 &
\end{tabular}

Fonte: elaborada com base nos resultados da pesquisa.

Quanto ao fator F2, verifica-se associação positiva entre as variáveis V10, V11, V12, V13, V14 e V15, refletindo indicadores relativos à dimensão ambiental. Esse fator busca destacar variáveis importantes para o desenvolvimento rural, no âmbito das questões envolvendo a degradação ambiental. Tal prática abusiva está associada ao uso de agrotóxicos, corretivos e incidência de queimadas. Segundo Lemos (2001), a ideia implícita é que, principalmente as lavouras (intensivas, sobretudo, as monoculturas) têm maior impacto ambiental por usarem práticas que devastam o meio ambiente, sendo que, neste estudo, o milho e o feijão foram escolhidos por serem as lavouras mais representativas no Ceará e em Pernambuco.

Por sua vez, o fator F3, que explica $12,18 \%$ da variância total dos dados, é constituído pelas variáveis V8 e V9. Segundo Kageyama (2004), esse fator reflete a dimensão demográfica, em que, quanto maior a população rural em relação à urbana, maior a capacidade da área rural reter a população, assim como a maior proporção de pessoas que vieram de outros municípios, havendo indícios da capacidade de atração que aquele município exerce na região.

\section{Índice de Desenvolvimento Rural (IDR)}

Com suporte na Tabela 03, é possível verificar que o desenvolvimento rural cearense é caracterizado por uma concentração expressiva em âmbito estadual, uma vez que apenas $4,65 \%$ dos municípios considerados, que correspondem a oito municípios (Acopiara, Canindé, Icó, Iguatu, Mauriti, Mombaça, Quixeramobim e Tauá), conseguiram alcançar IDR classificado como muito alto. De acordo com o 
DATASUS (2010), estes municípios ensejados destacaram-se pelo maior Valor Adicionado Bruto $(\mathrm{VAB})^{7}$, vis-à-vis aos demais.

Tabela 03- Frequência absoluta e relativa do Índice de Desenvolvimento Rural (IDR) dos municípios cearenses, 2010

\begin{tabular}{c|c|c}
\hline IDR & Frequência absoluta & Frequência relativa (\%) \\
\hline Muíssimo Alto & 01 & 0,58 \\
Muito Alto & 08 & 4,65 \\
Alto & 16 & 9,30 \\
Médio & 49 & 28,49 \\
Baixo & 71 & 41,28 \\
Muito Baixo & 26 & 15,12 \\
Muitíssimo Baixo & 01 & 0,58 \\
\hline TOTAL & 172 & 100,00
\end{tabular}

Fonte: elaborada com base nos resultados da pesquisa.

Reforçando essas constatações, destaca-se que somente o município de Boa Viagem foi classificado como muitíssimo alto IDR, podendo estar associado aos maiores valores nas variáveis referentes ao valor adicionado bruto $(R \$ 38.115,9)$ e área plantada de milho (2.272 ha.). Esse resultado sinaliza, segundo Kageyama (2008) e Stege (2011), que não necessariamente municípios com elevada produção agrícola conseguem atingir maior nível de desenvolvimento. Tal argumento é ressaltado com base nos dados do IPECE (2010), que mostra o maior PIB agrícola municipal cearense encontra-se no município de Tianguá ( $R \$ 82.028,00)$, não obstante classificado como médio desenvolvimento rural.

Portanto, os resultados são desfavoráveis, uma vez que parcela majoritária dos municípios (98) obtiveram IDR baixo, muito baixo ou muitíssimo baixo, que corresponde a $56,98 \%$ dos municípios cearenses analisados neste estudo. A esse respeito, ressalta-se que o município de Maracanaú, na mesorregião Metropolitana de Fortaleza, foi o único classificado com desenvolvimento rural muitíssimo baixo, podendo ser atribuído, sobretudo, às variáveis demográficas concernentes à proporção da população rural (\%) em relação à população total $(69,00 \%)$ e proporção de migrantes alocados nas zonas rurais (95,00\%). Segundo Lima Júnior (2014), este município possui elevado grau de urbanização, no qual está agregado à Região Metropolitana de Fortaleza (RMF), atuando como "polarizador" de atividades produtivas, especificamente do setor industrial e serviços.

\footnotetext{
7 Nessa perspectiva, a agricultura dos estados do Ceará e Pernambuco é responsável pelo Valor Adicionado Bruto (VAB) em termos relativos de 5,06\% e 4,78\%, respectivamente, participação que encobre a importância para a economia rural, como fonte de ocupação dos municípios do Nordeste, cujos produtos econômicos são dependentes de serviços nos quais a administração pública tem peso relevante. Em segundo lugar, parte das atividades do setor industrial, comercial e dos serviços está vinculada e dependente da agricultura. Portanto, há forte correlação entre a agricultura e os demais setores econômicos.
} 
Com base na Tabela 04, é possível verificar a expressiva heterogeneidade no desenvolvimento rural pernambucano, visto que apenas $3,17 \%$ dos municípios considerados conseguiram alcançar IDR classificados como muitíssimo alto ou muito alto. Em outros termos, apenas cinco municípios (Araripina, Buíque, Ouricuri, Petrolina e Santa Maria da Boa Vista) obtiveram resultados com essas classificações, sendo que somente dois deles (Petrolina e Araripina) foram incluídos na melhor escala de classificação, atingindo valores máximos referentes às variáveis: valor adicionado bruto agropecuário e número de pessoas alfabetizadas alocadas no meio rural, respectivamente. Esse resultado conquistado pelo município de Petrolina está consistente com o obtido por Lima e Sousa (2017).

Tabela 04- Frequência absoluta e relativa do Índice de Desenvolvimento Rural (IDR) dos municípios pernambucanos, 2010

\begin{tabular}{c|c|c}
\hline IDR & Frequência absoluta & Frequência relativa (\%) \\
\hline Muitíssimo Alto & 02 & 1,27 \\
Muito Alto & 03 & 1,90 \\
Alto & 19 & 12,03 \\
Médio & 45 & 28,48 \\
Baixo & 68 & 43,04 \\
Muito Baixo & 21 & 13,29 \\
Muitíssimo Baixo & 0 & 0,00 \\
\hline TOTAL & 158 & 100,00 \\
\hline
\end{tabular}

Fonte: elaborada com base nos resultados da pesquisa.

Por outro lado, embora nenhum município tenha registrado valores especificados como muitíssimo baixo, os resultados são insatisfatórios, uma vez que parcela expressiva dos municípios (68) obteve baixo IDR, que corresponde a $43,04 \%$ dos municípios pernambucanos analisados neste estudo. Ademais, outros 21 municípios $^{8}$ (Araçoiaba, Abreu e Lima, Arco Verde, Barra de Guaribara, Barreiros, Camutanga, Carpina, Condado, Cupira, Ferreiros, Itambé, Itaquitinga, Jaboatão dos Guararapes, Joaquim Nabuco, Lagoa do Itaenga, Moreno, Nazaré da Mata, Olinda, Santa Cruz do Capibaribe, Timbaúba e Tracunhaém) obtiveram resultados ainda piores, classificados como muito baixo.

A partir da Tabela 05, é possível verificar o IDR, em termos médios, para as mesorregiões cearenses e pernambucanas no ano de 2010. Os dados mostram que o Ceará possui IDR médio $(0,21)$ um pouco acima de Pernambuco $(0,18)$. No recorte

\footnotetext{
8 De acordo com os dados provenientes do IBGE (2010a), IBGE (2010b) e RAIS (2010), nesta amostra de municípios, os menores valores das variáveis analisadas foram registrados: Valor da produção das lavouras permanentes $(33,33 \%)$, Número de estabelecimentos agropecuários que utilizaram técnica de irrigação $(14,30 \%)$, Número de vínculos formais de trabalho no setor agropecuário (14,29\%), Proporção de migrantes de outros municípios $(14,29 \%)$, Área plantada de milho $(9,52 \%)$, Área plantada de feijão $(9,51 \%)$ e Proporção da população rural em relação à população total $(4,76 \%)$.
} 
mesorregional cearense, Sertões $(0,25)$, Centro-Sul $(0,24)$ e Sul $(0,22)$ obtiveram índices médios superiores ao Ceará. No caso das mesorregiões pernambucanas, São Francisco $(0,23)$, Sertão $(0,22)$ e Agreste $(0,19)$ alcançaram índices médios superiores à média estadual de Pernambuco.

Quanto ao Noroeste cearense, os maiores índices estão nos municípios de Viçosa do Ceará $(0,36)$ e Coreaú $(0,36)$, enquanto os piores resultados estão em Senador Sá $(0,09)$ e Varjota $(0,08)$. No Norte cearense, os maiores valores estão nos municípios de Canindé $(0,40)$ e Itapipoca $(0,35)$, entretanto seus piores valores estão em São Luís do Curu $(0,10)$ e Uburetama $(0,10)$. No tocante aos Sertões, os municípios de Boa Viagem $(0,47)$ e Tauá $(0,40)$ obtêm maiores IDR, enquanto Nova Russas $(0,13)$ e Saboeiro $(0,16)$ configuram os piores resultados.

Tabela 05- Índice de Desenvolvimento Rural médio das mesorregiões cearenses e pernambucanas para o ano de 2010

\begin{tabular}{c|c|c|c}
\hline $\begin{array}{c}\text { Mesorregiões } \\
\text { cearenses }\end{array}$ & IDR & $\begin{array}{c}\text { Mesorregiões } \\
\text { pernambucanas }\end{array}$ & IDR \\
\hline Noroeste & 0,20 & Sertão & 0,22 \\
Norte & 0,19 & São Francisco & 0,23 \\
Metropolitana & 0,12 & Agreste & 0,19 \\
Sertões & 0,25 & Zona da Mata & 0,12 \\
Jaguaribe & 0,20 & Metropolitana & 0,10 \\
Centro-Sul & 0,24 & & \\
Sul & 0,22 & & 0,18 \\
\hline CEARÁ & 0,21 & PERNAMBUCO &
\end{tabular}

Fonte: elaborada com base nos resultados da pesquisa.

Os municípios de Morada Nova $(0,37)$ e Russas $(0,34)$ destacaram-se com os maiores IDR da mesorregião Jaguaribe. Tal resultado pode ser decorrente dos perímetros irrigados ${ }^{9}$ contribuírem para geração de emprego e renda. Os municípios de Iracema $(0,11)$ e Fortim $(0,12)$, porém, tiveram os menores índices. Quanto ao Centro-Sul, os municípios de Icó $(0,42)$ e Iguatu $(0,38)$ atingiram maiores valores do IDR, enquanto que Baixio e Antonina apresentaram, 0,3 e 0,16, respectivamente, configurando-se como piores IDR.

Com relação ao Sul cearense, os piores índices ocorreram nos municípios de Altaneira $(0,09)$ e Penaforte $(0,11)$. Ao contrário, Mauriti $(0,38)$ e Missão Velha $(0,32)$ registraram os maiores IDR neste recorte geográfico. Verifica-se também que, em termos médios, a mesorregião Metropolitana de Fortaleza $(0,12)$ é inferior à média

9 O Perímetro Irrigado Tabuleiros de Russas está localizado nos municípios de Russas, Limoeiro do Norte e Morada Nova, mais precisamente no Baixo Vale do Jaguaribe e distante $160 \mathrm{~km}$ da capital cearense, Fortaleza. Produz frutas, hortaliças, grãos, pastagem, cana-de-açúcar, madeira (sabiá) e oleaginosas. Entre os benefícios esperados, têm-se: 15 mil empregos diretos, 30 mil empregos indiretos; 240 mil pessoas diretamente beneficiadas e $\mathrm{R} \$ 180$ mil por ano de renda (ADECE, 2011). 
do Ceará $(0,21)$, podendo ser explicada pela presença das atividades industriais, dada a proximidade com a capital. Os dados da RAIS (2010) mostram, que, do total de 172 municípios cearenses contemplados neste estudo, apenas oito (Aquiraz, Caucaia, Guaiúba, Maracanaú, Maranguape, Pacatuba, Horizonte e Pacajus) concentravam $32,19 \%$ dos estabelecimentos industriais do Estado.

Considerando as mesorregiões pernambucanas, os municípios de Araripina $(0,46)$ e Ouricuri $(0,41)$ no sertão pernambucano destacaram-se pelos maiores IDR, enquanto Arco Verde $(0,09)$ e Trindade $(0,11)$ apresentaram os menores valores. No tocante ao São Francisco, percebe-se que Petrolina $(0,58)$ e Santa Maria da Boa Vista $(0,40)$ obtiveram os maiores IDR, estando em consonância com o estudo de Lima e Sousa (2017).

Nessa perspectiva, segundo os dados DATASUS (2010), Petrolina possui o maior PIB agrícola nordestino ( $\mathrm{R} \$ 620.359,00)$, sendo que a expressiva participação absoluta de alfabetizados no meio rural (46.901 indivíduos) pode ser explicada pela taxa de analfabetismo rural municipal $(20,1 \%)$ menor que a média nordestina $(32,0 \%)$ e nacional $(22,9 \%)$.

$\mathrm{Na}$ mesorregião do Agreste, os municípios mais representativos quanto ao desenvolvimento rural são: Buíque $(0,35)$ e São Bento do Una $(0,34)$. Por outro lado, Santa Cruz do Capibaribe $(0,05)$ e Guaribara $(0,04)$ tiveram os menores índices. Considerando a Zona da Mata pernambucana, os municípios de Vicência $(0,21)$ e Quipapá $(0,20)$ destacaram-se pelos maiores IDR, ao passo que Carpina $(0,05)$ e Condado $(0,06)$ registraram os menores valores. No que se refere à mesorregião Metropolitana de Recife, percebe-se o índice médio $(0,10)$ inferior ao observado nos demais recortes geográficos de Pernambuco, podendo estar associado à maior densidade produtiva industrial, uma vez que, segundo a RAIS (2010), do total de 158 municípios pernambucanos congregados neste estudo, parcela minoritária de oito (Aracoiaba, Ilha de Itamaracá, Itapissuma, Abreu e Lima, Jaboatão dos Guararapes, Moreno, Olinda e Ipojuca) concentravam 21,66\% dos estabelecimentos industriais.

\section{Correlação entre as variáveis: IDR, PIB agrícola e população rural}

A matriz de correlações apresentada na Tabela 06 contém os coeficientes de correlação de Pearson entre as variáveis e os p-values para o Ceará e suas mesorregiões. Os resultados indicam que há uma correlação linear e positiva muito 
alta entre o IDR obtido neste estudo e a população rural, enquanto que o PIB agrícola sinalizou uma moderada correlação, seguindo a classificação de Bisqueira, Sarriera e Martínez (2004).

A mesorregião Centro-Sul foi a que aferiu maior correlação entre o IDR e o PIB agrícola $(0,89)$, classificando-se como muito alta, enquanto que a menor foi atribuída à mesorregião Noroeste $(0,56)$, sendo que a relação linear e positiva entre as duas variáveis consideradas para estes recortes geográficos é superior à correlação de Pearson para o Ceará, que se classificou como moderada $(0,40)$.

Tabela 06- Correlações entre o Índice de Desenvolvimento Rural (IDR) e as variáveis: PIB agrícola e população rural nas mesorregiões cearenses para o ano de 2010

\begin{tabular}{c|c|c|c|c|c|c|c|c}
\hline Discriminação & $\begin{array}{c}\text { Centro- } \\
\text { Sul }\end{array}$ & Jaguaribe & Metropolitana & Noroeste & Norte & Sertões & Sul & CE \\
\hline $\begin{array}{c}\text { Correlação de } \\
\text { Pearson entre } \\
\text { IDR e PIB } \\
\text { agrícola }\end{array}$ & $0,89^{*}$ & $0,61^{*}$ & $0,69^{* *}$ & $0,56^{*}$ & $0,58^{*}$ & $0,80^{*}$ & $0,65^{*}$ & $0,42^{*}$ \\
\hline Sig. (2-tailed) & 0,00 & 0,00 & 0,03 & 0,00 & 0,00 & 0,00 & 0,00 & 0,00 \\
\hline $\begin{array}{c}\text { Correlação de } \\
\text { Pearson entre } \\
\text { IDR e } \\
\text { população } \\
\text { rural }\end{array}$ & $0,94^{*}$ & $0,84^{*}$ & $0,95^{* *}$ & $0,71^{*}$ & $0,79^{*}$ & $0,93^{*}$ & $0,81^{*}$ & $0,70^{*}$ \\
\hline \begin{tabular}{c} 
Sig. (2-tailed) \\
\hline
\end{tabular} & 0,00 & 0,00 & 0,00 & 0,00 & 0,00 & 0,00 & 0,00 & 0,00 \\
\hline
\end{tabular}

Fonte: elaborada com base nos resultados da pesquisa.

*Significante a $1 \%$ (2-tailed); ${ }^{*}$ Significante a $5 \%$ (2-tailed).

No tocante à correlação entre o IDR e a população rural, para todas as mesorregiões analisadas, a relação linear entre essas duas variáveis mostrou-se superior ao PIB agrícola. O melhor desempenho foi verificado nas mesorregiões referentes à Metropolitana $(0,95)$ e Centro-Sul $(0,94)$, enquanto o menor resultado foi encontrado no Noroeste $(0,71)$, porém com valor um pouco acima da correlação para o Ceará $(0,70)$.

Segundo os resultados da Tabela 07, percebe-se que a mesorregião do São Francisco alcançou maior correlação entre o IDR e o PIB agrícola $(0,82)$, enquanto a menor foi à Metropolitana $(0,21)$. No tocante à correlação entre IDR e população rural, a mesorregião do São Francisco também liderou a maior correlação entre as variáveis $(0,87)$, ao passo que a Metropolitana registrou a menor correlação $(0,36)$.

Tabela 07- Correlações entre o Índice de Desenvolvimento Rural (IDR) e as variáveis: PIB agrícola e População Rural nas pernambucanas para o ano de 2010

\begin{tabular}{c|c|c|c|c|c|c}
\hline Discriminação & Agreste & $\begin{array}{c}\text { Zona da } \\
\text { Mata }\end{array}$ & Metropolitana & São Francisco & Sertão & PE \\
\hline $\begin{array}{c}\text { Correlação de } \\
\text { Pearson entre } \\
\text { IDR e PIB }\end{array}$ & $0,32^{* *}$ & $0,37^{\star}$ & $0,21^{* *}$ & $0,82^{*}$ & $0,46^{*}$ & $0,43^{\star}$ \\
\hline
\end{tabular}




\begin{tabular}{c|c|c|c|c|c|c}
\hline agrícola & & & & & & \\
\hline Sig. (2-tailed) & 0,04 & 0,00 & 0,02 & 0,00 & 0,00 & 0,00 \\
\hline $\begin{array}{c}\text { Correlação de } \\
\text { Pearson entre } \\
\text { IDR e } \\
\text { população } \\
\text { rural }\end{array}$ & $0,58^{* *}$ & $0,67^{*}$ & $0,36^{* *}$ & $0,87^{*}$ & $0,80^{*}$ & $0,71^{*}$ \\
\hline Sig. (2-tailed) & 0,00 & 0,00 & 0,00 & 0,00 & 0,02 & 0,00 \\
\hline
\end{tabular}

Fonte: elaborada com base nos resultados da pesquisa.

${ }^{*}$ Significante a $1 \%$ (2-tailed); ${ }^{* *}$ Significante a $5 \%$ (2-tailed).

Esses resultados para os municípios cearenses e pernambucanos estão convergentes com os observados nos estudos de Kageyama (2004; 2008), Stege (2011) e Pinto e Coronel (2016) ao apontar que os melhores resultados para o desenvolvimento rural foram obtidos por municípios com maior população rural.

\section{Considerações Finais}

Os resultados mostraram que o fator F1 explica a maior variância $(32,62 \%)$ dos dados e reflete a dimensão socioeconômica. Quanto ao fator F2, verifica-se a segunda maior variância $(31,21 \%)$ e capta a dimensão ambiental, enquanto o fator F3 explica $12,18 \%$ da variância total dos dados e traduz a dimensão demográfica. Esses três fatores são responsáveis por $76 \%$ da variância total dos dados, sinalizando a importância de se investir nesses fatores como forma de melhorar o desenvolvimento rural, especialmente naqueles municípios cearenses e pernambucanos que mais carecem das variáveis que compõem tais dimensões.

Verifica-se uma expressiva heterogeneidade no desenvolvimento rural, em que, dos 330 municípios analisados, apenas nove (Acopiara, Boa Viagem, Canindé, Icó, Iguatu, Mauriti, Mombaça, Tauá e Quixeramobim) no Ceará e cinco (Araripina, Buíque, Ouricuri, Petrolina e Santa Maria da Boa Vista) em Pernambuco alcançaram IDR muito alto ou muitíssimo alto, o que representa apenas $4,24 \%$ do total dos municípios analisados.

Rastreando os dados processados, infere-se que tais municípios cearenses foram os que tiveram melhor desempenho quanto às variáveis ambientais consideradas neste estudo. Tais variáveis também colaboraram para o notório resultado do IDR obtido pelos municípios pernambucanos de Araripina, Buíque, Ouricuri, ao passo que Petrolina e Santa Maria da Boa Vista tiveram destaque nas variáveis sociais e econômicas. Em contrapartida, parcela expressiva de 186 municípios, o que equivale a $56,36 \%$, ostentaram IDR baixo ou muito baixo. Portanto, parcela majoritária dos municípios cearenses e pernambucanos se 
configurou com baixo IDR, o que agrava a qualidade de vida desse contingente populacional, contribuindo para o círculo vicioso da pobreza rural, uma vez que se verifica a concentração dos melhores resultados concernentes às condições sociais, econômicas, ambientais e demográficas em uma parcela minoritária dos municípios.

Assim, pode-se inferir que o desenvolvimento rural deve ser compreendido pelas múltiplas dimensões: socioeconômicas, ambientais e demográficas. Nessa perspectiva, é importante que os gestores municipais busquem traçar medidas de políticas públicas que acelerem o desenvolvimento rural, uma vez que os municípios não devem ser administrados de forma padronizada em virtude das especificidades de cada um, contribuindo para melhoria do bem-estar das populações rurais.

De posse dos resultados obtidos, constata-se ainda que os municípios com maiores PIBs agrícolas e população rural são os que promovem o maior desenvolvimento rural, uma vez que a população rural se mostrou positiva e fortemente correlacionada com o IDR, enquanto o PIB agrícola indicou uma moderada correlação linear. Esses resultados estão condizentes com a literatura.

Este estudo restringiu-se a um período específico, não podendo ser comparado com anos posteriores, dada a ausência de dados secundários que contemplem as variáveis deste estudo. Sugere-se que trabalhos futuros possam ampliar o recorte geográfico do estudo para outras áreas do Nordeste, haja vista que esta macrorregião congrega a maior população rural do Brasil.

\section{REFERÊNCIAS}

ADECE - AGÊNCIA DE DESENVOLVIMENTO DO ESTADO DO CEARÁ. Perímetros públicos irrigados do Ceará em 2011. Disponível em: <http:// www.adece.gov.br.agronegócios>. Acesso em: 10/05/2018.

ARAÚJO, T. B. Nordeste, Nordestes. Que Nordeste? Ensaios sobre o Desenvolvimento Brasileiro: heranças e urgências. Rio de Janeiro: Fase, 2000.

BEGNINI, S.; ALMEIDA, L. E. D. F. Desenvolvimento rural no estado de Santa Catarina um estudo multidimensional. Gestão \& Regionalidade (Online), v. 32, n. 94, p.20-35, jan./abr. 2016.

BISQUEIRA, R.; SARRIERA, J. C.; MARTÍNEZ, F. Introdução à Estatística: enfoque informático com o pacote estatístico SPSS. Porto Alegre: Atmed, 2004.

CANO, W. Ensaios sobre a crise urbana no Brasil. Campinas: Editora da UNICAMP, 2011. 
COELHO, A. S. N. Trajetórias e direcionamentos da política de irrigação no Brasil: as especificidades da região nordeste e do vale do São Francisco. In: Encontro Nacional de Geografia, 19, 2009. Anais... São Paulo: ENG, 2009.

DALCHIAVON, F. C.; CARVALHO, M.P. Correlação linear e espacial dos componentes de produção e produtividade da soja. Ciências Agrárias (Online), Londrina, v. 33, p. 541-552, abr. 2012.

DATASUS - DEPARTAMENTO DE INFORMÁTICA DO SUS. 2010. Disponível em: <http://www2.datasus.gov.br/DATASUS/index.php?area=01 >. Acesso em 06/09/2017.

FÁVERO, L. P.; BELFIORE, P.; SILVA, F. L.; CHAN, B. L. Análise de dados: modelagem multivariada para tomada de decisões. Primeira edição. Rio de Janeiro. Elsevier, 2009.

FURTADO, C. Formação econômica do Brasil. 17ª̣ edição. São Paulo: Editora Nacional, 1980.

GRISA, C. SCHNEIDER, S. Três gerações de políticas públicas para agricultura familiar e formas de integração entre sociedade e Estado no Brasil. In: GRISA, C.; SCHNEIDER, S. (Orgs.). Políticas públicas de desenvolvimento rural no Brasil. Porto Alegre: Editora da UFRGS, 2015.

HAIR JÚNIOR, J. F.; BLACK, W. C.; BABIN, B. J.; ANDERSON, R. E.; TATHAM, R. L. Análise multivariada de dados. 6.ed. Porto Alegre: Bookman, 2009.

HELFAND, S.; PEREIRA, V. Determinantes da pobreza rural e implicações para as políticas públicas no Brasil. In: BUAINAIN, A. M; DEDECCA, C. S. (Org.). A nova cara da pobreza rural: desafios para as políticas públicas. Brasília: IICA, Série Desenvolvimento Rural Sustentável, v. 16, p. 121-160, 2012.

IBGE - INTITUTO BRASILEIRO DE GEOGRAFIA E ESTATÍSTICA. Censo Agropecuário 2006-2007. 2010a. Disponível em: <www.ibge.gov.br>. Acesso em: 02/06/2017.

IBGE - INTITUTO BRASILEIRO DE GEOGRAFIA E ESTATÍSTICA. Censo Demográfico de 2010. 2010b. Disponível em: <www.ibge.gov.br>. Acesso em: 05/06/2017.

IPECE - INSTITUTO DE PESQUISA E ESTRATÉGIA ECONÔMICA. 2010. Disponível em: $<w w w . i p e c e . c e . g o v . b r>$. Acesso em: 08/03/ 2018.

JESUS, C. M. Desenvolvimento Territorial Rural: análise comparada entre os territórios constituídos autonomamente e os induzidos pelas políticas públicas no Brasil e Espanha. Uberlândia, MG, 215p. Tese (Doutorado em Políticas Públicas e Desenvolvimento) Universidade Federal de Uberlândia, 2013.

KAGEYAMA, A. Desenvolvimento rural: conceito e medida. Cadernos de Ciência \& Tecnologia, v. 21, n. 3, p. 379-408, set./dez. 2004.

KAGEYAMA, A. Desenvolvimento rural: conceitos e aplicações ao caso brasileiro. Porto Alegre: Editora da UFRGS, 2008.

LEMOS, J. J. S. Níveis de degradação no Nordeste Brasileiro. Revista Econômica do Nordeste, Fortaleza, v. 32, n. 3, p. 406-429, jul./set. 2001.

LEMOS, M. B. Espaço e capital: um estudo sobre a dinâmica centro x periferia. Campinas, SP, 293 p. Tese (Doutorado em Economia) - Universidade Estadual de Campinas, 1988. 
LIMA JÚNIOR, F. O'. Estrutura produtiva e rede urbana no estado do Ceará durante o período de 1980-2010. Campinas, SP, 264 p. Tese (Doutorado em Desenvolvimento Econômico) - Universidade Estadual de Campinas, 2014.

LIMA, R. J.; SOUSA, E. P. Desenvolvimento rural dos municípios da Região Integrada Petrolina (PE) - Juazeiro (BA). Cadernos de Ciências Sociais Aplicadas (UESB), Vitória da Conquista, v. 14, p. 1-18, 2017.

MELO, C. O.; PARRÉ, J. L. Índice de desenvolvimento rural dos municípios paranaenses: determinantes e hierarquização. Revista de Economia e Sociologia Rural, Brasília, v. 45, n. 2, p. 25-53, abr./jun. 2007.

MINGOTI, S. A. Análise de dados através de métodos de estatística multivariada: uma abordagem aplicada. Belo Horizonte: Editora da UFMG, 2005.

NAVARRO, Z. Desenvolvimento rural no Brasil: os limites do passado e os caminhos do futuro. Estudos Avançados, São Paulo, v. 15, n. 43, p. 83-100, set./dez. 2001.

PINTO, N. G. M.; CORONEL, D. A. Desenvolvimento rural no Rio Grande do Sul: uma análise das mesorregiões entre o período de 2000 e 2010. Ensaios FEE, Porto Alegre, v. 36, n. 4, p. 893 - 920, mar. 2016.

POLASTRINI, A. C.; MARTINS, C. L.; TREDEZINI, C. A. D. Indicadores de desenvolvimento rural: análise comparativa entre os territórios CONSADS da região Centro-Oeste e Nordeste. Sociedade e Desenvolvimento Rural, v. 9, p.73-99, nov. 2015.

RAIS - RELAÇÃO ANUAL DE INFORMAÇÕES SOCIAIS. 2010. Disponível em: <http//www.mte.gov.br>. Acesso em: 28/07/ 2017.

REZENDE, L. P.; PARRÉ, J. L. A regionalização da agricultura paranaense na década de 1990: um estudo utilizando estatística multivariada. In: Congresso Brasileiro de Economia, Administração e Sociologia Rural, 42, 2004. Anais... Cuiabá, MT: SOBER, 2004.

SABINO, M. J. C. A vulnerabilidade da agricultura familiar nos munícipios do Ceará: o caso do Maciço de Baturité. Fortaleza, CE, 95 p. Dissertação (Mestrado em Economia Rural) - Universidade Federal do Ceará, 2013.

SAleS, A. P.; RODRIGUES, C. P. G.; BARRETO, A. C.; ALMEIDA, M. R. D. O desenvolvimento rural dos municípios do Ceará. In: Colóquio Sociedade, Políticas Públicas, Cultura e Desenvolvimento, 6, 2017. Anais... Crato, CE: CEURCA 2017.

SANTINI, G. A.; OLIVEIRA, S. C.; PIGATTO, G. Análise da relação das variáveis preço e produção da mandioca tipo indústria no estado de São Paulo, 1996 a 2008. Informações Econômicas, São Paulo: IEA, v. 40, p. 41-52, mar. 2010.

SANTOS, L. F.; FERREIRA, M. A. M.; SALGADO, R. F. S. F. Desenvolvimento rural nos municípios de Minas Gerais: fatores determinantes e hierarquização. Gestão \& Regionalidade (Online), v.33, n. 97, p.88-99, jan./abr. 2017.

SILVA, M. M. A. S.; LIMA, P. V. P. S. Indicadores educacionais e de renda e sua relação com pobreza e indigência no Semiárido Brasileiro. Revista Econômica do Nordeste, Fortaleza, v. 46, p. 117-132, abr./jun. 2015.

STEGE, A. L. Desenvolvimento rural nas microrregiões do Brasil: um estudo multidimensional. Maringá, PR, 137 p. Dissertação (Mestrado em Economia) - Universidade Estadual de Maringá, 2011. 
TRIOLA, M F. Introdução à Estatística. 11 ed. Rio de Janeiro: LTC, 2013.

\section{NOTAS DE AUTOR}

\section{CONTRIBUIÇÃO DE AUTORIA}

José Ediglê Alcantara Moura - Concepção. Coleta de dados. Análise de dados. Elaboração do manuscrito, revisão e aprovação da versão final do trabalho.

Eliane Pinheiro de Sousa - Concepção e elaboração do manuscrito. Participação ativa da discussão dos resultados. Revisão e aprovação da versão final do trabalho.

\section{FINANCIAMENTO}

Não se aplica.

\section{CONSENTIMENTO DE USO DE IMAGEM}

Não se aplica.

APROVAÇÃO DE COMITÊ DE ÉTICA EM PESQUISA

Não se aplica.

\section{CONFLITO DE INTERESSES}

Não se aplica.

\section{LICENÇA DE USO}

Este artigo está licenciado sob a Licença CreativeCommons CC-BY. Com essa licença você pode compartilhar, adaptar, criar para qualquer fim, desde que atribua a autoria da obra.

\section{HISTÓRICO}

Recebido em: 04-09-2019

Aprovado em: 13-07-2020 